\title{
Un nuevo medio de cultivo líquido para el patógeno Piscirickettsia salmonis
}

\author{
A new liquid medium for the pathogen Piscirickettsia salmonis
}

\author{
T Vera*, A Isla, A Cuevas, J Figueroa \\ Laboratorio de Biología Molecular de Peces, Instituto de Bioquímica y Microbiología, Facultad de Ciencias,
} Universidad Austral de Chile, Valdivia, Chile.

\begin{abstract}
SUMMARY
Piscirickettsia salmonis is the ethiologic agent of the Salmonid Rickettsial Syndrome (SRS), which was first described in cultured Coho salmon in the south coast of Chile in 1989 and since then has been reported in different places around the world. Initially, this bacterium was described as an obligate intracellular pathogen, able to grow only in cytoplasmic vacuoles in host cell where it replicates by binary fission, so their in vitro culture is performed mainly in cell lines derived from fish. Recently, it has been demonstrated that this bacterium can grow in cell-free media containing sheep blood or fish and high levels of cysteine; however, due to the presence of cellular elements these culture media present drawbacks regarding handling, storage and high costs. We propose an artificial culture medium supplemented with an iron salt to replace the blood that is traditionally used; it reported successful growth of the bacterial reference strain and of a strain isolated from the environment, which was evaluated by optical density (OD $\left.{ }_{600}\right)$, while purity and identity of the culture were checked by using Gram stain, immunofluorescence and PCR. Developing a blood-free culture medium facilitates the cultivation of $P$. salmonis and allows the growth of this bacterium in a liquid medium, which provides various microbiological and biotechnological applications.
\end{abstract}

Palabras clave: cultivo líquido, Piscirickettsia salmonis.

Key words: liquid culture, Piscirickettsia salmonis.

\section{INTRODUCCIÓN}

Piscirickettsia salmonis es el agente etiológico del síndrome ricketssial salmonídeo (SRS), causante de una agresiva infección sistémica en especies salmonídeas de varias partes del mundo (Bravo y Campos 1989, Rojas y col 2009), siendo Chile uno de los países más afectados donde ha causado importantes pérdidas económicas para la industria salmonera (McCarthy y col 2008). La primera descripción de este microorganismo fue realizada a partir de aislados obtenidos desde cultivos de salmón Coho (Oncorhynchus kisutch) en el sur de Chile en 1989. Desde entonces, la presencia del patógeno y la enfermedad han sido reportadas en las costas de Irlanda (Rodger y Drinan 1993), Noruega (Olsen y col 1997), Escocia (Birrell y col 2003), Tasmania (Corbeil y col 2005), costa atlántica de Canadá (Cusack y col 2002), y la costa del océano Pacífico de Canadá (Brocklebank y col 1992). Además, la presencia del patógeno ha sido reportada en otras especies de peces no salmonídeos de interés comercial como lubina o European Seabass (Dicentrarchus labrax) (McCarthy y col 2005), corvina blanca o White Seabass (Atractoscion nobilis) (Arkush y col 2005) y en poblaciones de Tilapia (Oreochromis mossambicus y Sarotherodon melanotheron) (Athanassopoulou y col 2004).

Aceptado: 19.04.2012.

* Casilla 567, Valdivia, Chile; veratamara@gmail.com
P. salmonis es una bacteria gram negativa, inmóvil, sin cápsula, pleomórfica, pero generalmente cocoide con un diámetro que varía entre 0,2 y 1,5 $\mu \mathrm{m}$ (Bravo y Campos 1989, Fryer y col 1990, Rojas y col 2009). Inicialmente se describió como un patógeno intracelular obligado, replicando en vacuolas citoplasmáticas tanto in vivo como en líneas celulares derivadas de peces (Fryer y col 1990, Garcés y col 1991, Almendras y col 1997, Mauel y Miller 2002, McCarthy y col 2008). Originalmente, el nombre de la bacteria fue atribuido a ciertas similitudes con especies rickettsiales, tales como la replicación en inclusiones citoplasmáticas en células hospederas y pared celular ondulada. Sin embargo, análisis posteriores de la cepa tipo LF-89 la sitúan dentro de la subdivisión gamma de la clase proteobacteria, a la cual pertenecen los géneros Coxiella, Legionella y Francisella y no en la subdivisión alpha a la cual pertenece el género Rickettsia (Fryer y col 1992, Fryer y Hedrick 2003).

Tradicionalmente, este patógeno es propagado en varias líneas de células derivadas de peces, tales como CHSE 214 (Chinook salmon embryo cells), SHK1 (Salmon head kidney), EPC (Epithelioma papulosum cyprini), BB (Brown bullhead catfish), RTS11 (Rainbow trout spleen), donde su crecimiento es controlado, provocando la aparición gradual de efecto citopático (Fryer y col 1990, Almendras y col 1997, Almendras y Fuentealba 1997, Rojas y col 2009). No obstante, reportes de Mikalsen y col 2008 y Mauel y col 2008 entregan evidencia sobre la naturaleza intracelular facultativa del microorganismo, indicando la 
utilización del medio sólido Agar-Sangre suplementado con Suero Bovino Fetal (SBF), Glucosa y L-Cisteína (L-Cys), destacando que esta última es esencial para el crecimiento del microorganismo en medio sólido.

El desarrollo de medios de cultivo libres de células, utilizando sangre de ovino o peces, disminuye los costos asociados con el manejo de este microorganismo en líneas celulares y facilita su aislamiento y transporte para los ensayos de diagnóstico (Mauel y col 2008, Mikalsen y col 2008). Sin embargo, la utilización de sangre también presenta inconvenientes asociados con el costo, condiciones de almacenamiento y el manejo en la preparación de las placas de agar. A pesar de ello, el único antecedente de un medio de cultivo líquido para $P$. salmonis corresponde a un medio suplementado con sangre de peces, el cual presentó una densidad óptica máxima de 0,25 OD $_{620}$ después de 13 días de incubación; dicho valor es muy bajo con respecto a otros microorganismos como Escherichia coli, lo que estaría directamente relacionado con el agotamiento de algún nutriente limitante (Gómez y col 2009). Debido a que no existen otros antecedentes relacionados con medios de cultivo líquido o sólidos libres de elementos celulares para $P$. salmonis, el presente trabajo expone el desarrollo de un medio de cultivo para el crecimiento de este microorganismo. Se propone la utilización de una sal de hierro en substitución de la sangre utilizada en los medios de cultivo debido a que el hierro es uno de los principales componentes de la hemoglobina. Además, trabajos desarrollados en otros patógenos intracelulares como Mycobacterium tuberculosis y Salmonella typhimurium indican que el hierro es esencial para la sobrevivencia intracelular en macrófagos, crecimiento, multiplicación y manifestación de la enfermedad, siendo un elemento esencial para los microorganismos (Collins 2003, Ratledge 2004). Con estos antecedentes postulamos en el presente artículo un nuevo medio de cultivo para el patógeno intracelular facultativo $P$. salmonis.

\section{MATERIAL Y MÉTODOS}

\section{CULTIVO EN MEDIO LÍQUIDO}

Para probar si $P$. salmonis crecía en medio líquido se tomaron $5 \mathrm{ml}$ de sobrenadante de cultivo celular SHK1 infectado con P. salmonis, con 80-90\% de efecto citopático visible; se centrifugó a 300 x $g$ para eliminar debri celular. El sobrenadante se concentró a 4.000 x $g$ durante $10 \mathrm{~min}$, el sedimento se resuspendió en $5 \mathrm{ml}$ de un caldo conteniendo peptona de caseína $17,0 \mathrm{~g} / 1$, peptona de harina de soya 3,0 g/l, D (+) glucosa 2,5 g/l, NaCl 20,0 g/l, fosfato monoácido de dipotasio 2,5 g/l (Caldo A), el cual fue suplementado con L-Cys 0,1\%, SBF 2,5\%, $\mathrm{FeCl}_{3} 10 \mathrm{mg} / \mathrm{l}$, $\mathrm{NaCl} 15 \mathrm{~g} / \mathrm{l}$. Los cultivos fueron incubados a $17^{\circ} \mathrm{C}$ con agitación suave; el cultivo se evaluó periódicamente al medir $\mathrm{DO}_{600}$ durante 10 días para construir la curva de crecimiento.

\section{CULTIVO EN MEDIO SÓLIDO}

El Caldo A, con agar agar a $15 \mathrm{~g} / \mathrm{l}$ y $\mathrm{NaCl} 15 \mathrm{~g} / \mathrm{l}$ se esterilizó por autoclave y se suplementó con L-Cys $1 \mathrm{~g} / \mathrm{l}$, $\mathrm{FeCl}_{3} 20 \mathrm{mg} / \mathrm{l}$, SBF $5 \%$. Los cultivos se incubaron a $17^{\circ} \mathrm{C}$ durante 12 días.

\section{PCR ANIDADO PARA DETECCIÓN}

La detección de $P$. salmonis por PCR se realizó según lo descrito por Mauel y col en 1996.

\section{EXTRACCIÓN DE DNA GENÓMICO}

La extracción del DNA genómico se realizó con Wizard Genomic DNA purification Kit (Promega), según las instrucciones del proveedor.

\section{ANÁLISIS CITOLÓGICO}

Se realizaron extendidos de cultivo líquido y sólido a los que se les hizo tinción de gram, y posteriormente sobre la misma muestra se efectuó inmunofluorescencia indirecta (IFAT) con SRS fluoro-test-indirecto (Bioschile), según las indicaciones del proveedor.

\section{RESULTADOS Y DISCUSIÓN}

El crecimiento en el medio líquido propuesto se evaluó midiendo la $\mathrm{DO}_{600}$ durante 12 días, alcanzando $\mathrm{DO}_{600}$ de 1,8 (figura 1A). Para comprobar la pureza del cultivo líquido y que el aumento de la densidad óptica corresponde con un incremento en el número de microorganismo se tomaron muestras en diferentes tiempos y se realizó tinción de gram e IFAT en el mismo frotis. Los resultados indican que las bacterias mantienen su forma cocoide característica, propiedades de tinción y tamaño acordes a lo descrito previamente. Además, se observó un aumento en el número de microorganismos en las diferentes fases de crecimiento microbiano (figura 1C). El análisis de la curva de crecimiento arrojó un tiempo de generación estimado en 12,4 h.

La confirmación definitiva de la identidad de esta bacteria se obtuvo al realizar PCR anidado. En una primera ronda de PCR con cebadores para el 16S de eubacterias se obtiene un producto de amplificación de 1505 pb y una segunda ronda de amplificación con cebadores específicos para $P$. salmonis, se obtiene un producto de amplificación de $467 \mathrm{pb}$ (figura 2).

Habiendo conseguido con éxito la formulación de un medio líquido alternativo a los descritos hasta el momento, se destaca en primer lugar el uso de $\mathrm{FeCl}_{3}$ como fuente de hierro reemplazando la adición de sangre en el medio de cultivo. La utilización de sales de hierro como $\mathrm{Fe}\left(\mathrm{NO}_{3}\right)_{3}, \mathrm{FeSO}_{4}$ logra un resultado similar al mostrado utilizando $\mathrm{FeCl}_{3}$ como fuente de hierro (dato no mostrado), 

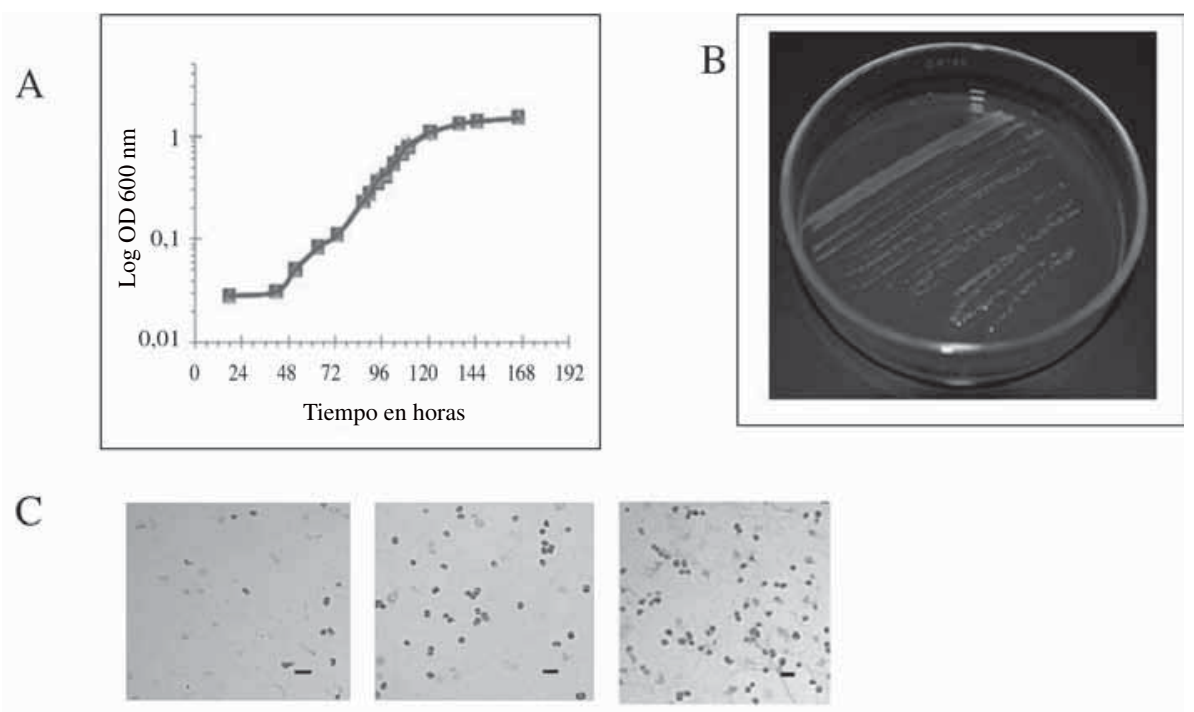

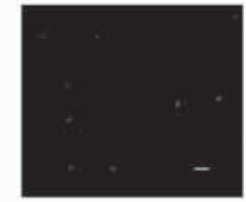

Fase latencia

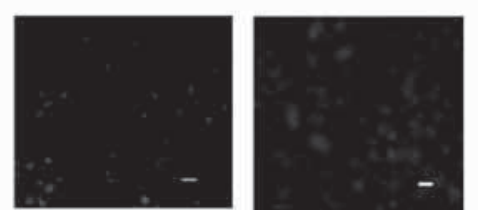

Fase exponencial

Figura 1. Crecimiento de P. salmonis cepa LF-89 en medio líquido y sólido. (A) Curva de crecimiento en medio líquido realizada en cuadruplicado. (B) Crecimiento en placa de agar al día 12 de incubación a $17^{\circ} \mathrm{C}$. (C) Comprobación del incremento del número de bacterias en medio líquido. Tinción de gram e inmunofluorescencia indirecta en muestras tomadas desde la fase de latencia y crecimiento exponencial. Barra $2 \mu \mathrm{m}$.

P. salmonis LF-89 strain growth in liquid and solid medium. (A) Growth curve in liquid medium performed in quadruplicate. (B) Growth in agar plate at day 12 of incubation at $17^{\circ} \mathrm{C}$. (C) Verification of the increased in number of bacteria in liquid medium. Gram staining and indirect immunofluorescence in samples taken from the lag and exponential phase. Bar $2 \mu \mathrm{m}$.

indicando que $P$. salmonis utilizaría el hierro presente en el medio de cultivo independiente de su fuente de origen. La utilización de $\mathrm{Fe}^{3+} \mathrm{o} \mathrm{Fe}{ }^{2+}$ en los medios de cultivo fue descrita por Bridson y Brecker (1970), quienes describieron que la presencia de bajas concentraciones de iones usualmente estimula el crecimiento de los microorganismos. La necesidad de obtener hierro para el crecimiento de microorganismos patógenos intracelulares se ha descrito en M. tuberculosis, que han desarrollado mecanismos para capturar selectivamente el hierro presente en el medio (Collins 2003).

Durante el desarrollo de este nuevo medio de cultivo P. salmonis demostró ser capaz de crecer en el medio líquido sin suplementar con SBF, resultados similares a los obtenidos con los medios artificiales reportados para este microorganismo por Mauel y col en el año 2008. En este medio de cultivo carente de SBF, el patógeno $P$. salmonis aumenta la fase de latencia de manera considerable (dato no mostrado). Esto se debería a que el SBF entregaría factores de crecimiento necesarios durante la etapa de adaptación del microorganismo en el medio líquido (Altas 2010).
Una vez alcanzados los objetivos principales del desarrollo de un nuevo medio líquido para el cultivo de $P$. salmonis, el paso a seguir fue producir un medio sólido, el cual consiste en la misma formulación del medio líquido, salvo que se agregó 1,5\% agar-agar y se utilizó el doble de la concentración de $\mathrm{FeCl}_{3}$ y SBF, en donde se observó crecimiento bacteriano al día 4 y la aparición de colonias únicas se visualizó a los 9 días de incubación a $17{ }^{\circ} \mathrm{C}$ (figura $1 \mathrm{~B}$ ), verificando la pureza de los cultivos a través de tinción de gram, IFAT y PCR anidado para detección. Tanto en el medio líquido como sólido se sembraron, además de la cepa LF-89, diferentes cepas de $P$. salmonis que fueron obtenidas de distintos brotes y provistas por las empresas (Marin-Harvest y Aquagestion, Puerto Montt, Chile), obteniendo un crecimiento similar en todas ellas. Además, demostraron mantener su capacidad de infección al sembrarlas en cultivos de células (SHK-1), luego de reiterados pasajes tanto por medios líquidos como sólidos (dato no mostrado). Resultados similares fueron obtenidos en los medios artificiales suplementados con sangre (Gómez y col 2009). Esto indicaría que el nuevo medio 


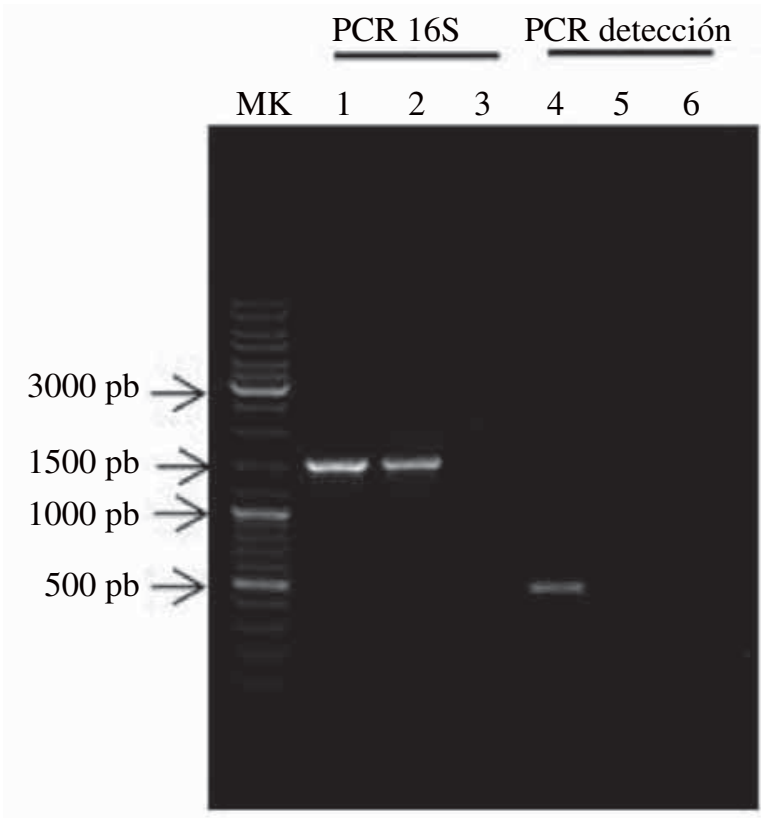

Figura 2. Verificación molecular de la pureza e identidad de los cultivos. Análisis por PCR al día 5 de crecimiento. MK gene ruler ladder mix (Fermentas). Líneas 1, 4 LF89, líneas 2, 5 E. coli, líneas 3 y 6 control negativo PCR anidado, primera y segunda ronda.

Molecular verification of the purity and identity of cultures. PCR analysis on day 5 of growth. MK gene ruler ladder mix (Fermentas). Lines 1, 4 LF89, Lines 2,5 E. coli, lines 3 y 6 negative control nested PCR, first and second round.

de cultivo líquido y sólido presentado en este trabajo no afectaría la capacidad de infección del microorganismo, permitiendo su utilización en investigación y diagnóstico.

Los resultados obtenidos indican la obtención de un nuevo medio de cultivo para el patógeno $P$. salmonis, permitiendo reducir las dificultades y costos implicados en la utilización de elementos celulares en los medios de cultivo para microorganismos, facilitando el manejo de $P$. salmonis en condiciones de laboratorio y en el trabajo de campo. Además permite cuantificar de manera rápida y sencilla la cantidad de bacterias presentes en el cultivo, lo cual es una gran ventaja para realizar ciertos estudios que utilicen la medición de este parámetro tales como el cálculo del Tiempo de Generación, la realización de antibiogramas cuantitativos (Forbes y col 1998) y aplicaciones biotecnológicas como una herramienta que facilitaría la investigación en el diseño de vacunas.

\section{RESUMEN}

Piscirickettsia salmonis es el agente causal del Síndrome Rickettsial Salmonídeo (SRS), el cual se describió por primera vez en cultivos de salmón Coho en las costas del Sur de Chile en el año 1989 y desde entonces se ha reportado en distintos lugares alrededor del mundo. Inicialmente esta bacteria se describió como un patógeno intracelular obligado, capaz de crecer sólo dentro de vacuolas citoplasmáticas de la célula hospedera en donde se replica por división binaria, por lo que su cultivo in vitro se realiza principalmente en líneas de células derivadas de peces. Actualmente se ha demostrado que esta bacteria es capaz de crecer en medios libres de células, conteniendo sangre de oveja o peces y altos niveles de cisteína. Sin embargo, estos medios de cultivo cuentan con la presencia de elementos celulares, lo que presenta inconvenientes de manejo, almacenamiento y costos elevados. Por este motivo se propone un medio de cultivo artificial suplementado con una sal de hierro en reemplazo de la sangre utilizada tradicionalmente. En el presente trabajo se logró un exitoso crecimiento bacteriano de la cepa tipo y cepas de campo el que fue evaluado por densidad óptica $\left(\mathrm{DO}_{600}\right)$; la pureza e identidad del cultivo fueron comprobadas por tinción de gram, inmunofluorescencia y PCR. La formulación de un medio de cultivo libre de sangre facilita el cultivo de $P$. salmonis y permite el crecimiento de esta bacteria en medio líquido, el cual facilita diversas aplicaciones microbiológicas y biotecnológicas.

\section{REFERENCIAS}

Almendras FE, IC Fuentealba. 1997. Salmonid rickettsial septicemia caused by Piscirickettsia salmonis: a review. Dis Aquat Org 29, 137-144.

Almendras FE, SRM Jones, IC Fuentealba, GM Wright. 1997. In vitro infection of a cell line from Ictalurus nebulosus with Piscirickettsia salmonis. Can J Vet Res 6, 66-68.

Altas R. 2010. Handbook of microbiological media. $4^{\text {th }}$ ed. CRC Press, Boca Raton, Florida, USA.

Arkush KD, AM McBride, HL Mendonca, MS Okihiro, KB Andree, S Marshall, V Henríquez, RP Hedrick. 2005. Genetic characterisation and experimental pathogenesis of Piscirickettsia salmonis isolated from white seabass Astractoscion nobilis. Dis Aquat Org 63, 139-49.

Athanassopoulou F, D Groman, T Prapas, O Sabatakou. 2004. Pathological and epidemiological observations on rickettsiosis in cultured sea bass (Dicentrarchus labrax L.) from Greece. J Appl Ichthyol 20, 525-529.

Birrell J, S Mitchell, DW Bruno. 2003. Piscirickettsia salmonis in farmed Atlantic salmon, Salmo salar in Scotland. B Eur Assoc Fish Pat 23, 213-218.

Bravo S, M Campos. 1989. Coho salmon syndrome in Chile. FHS/AMS Newsletter 17, 3 .

Bridson E, Y Brecker, 1970. Design and formulation of microbial culture media. In: Norris JR, Ribbons DW (eds). Methods in microbiology. Vol. 3A. Academic Press, New York, USA, Pp 274.

Brocklebank JR, DJ Speare, RD Armstrong, TP Evelyn. 1992. Septicemia suspected to be caused by a rickettsia-like agent in farmed Atlantic salmon. Can Vet J 33, 407-408.

Collins H. 2003. The role of iron in infections with intracellular bacteria. Immunol Lett 85, 193-195.

Corbeil S, AD Hyatt, MSJ Crane. 2005. Characterization of an emerging rickettsia-like organism in Tasmanian farmed Atlantic salmon Salmo salar. Dis Aquat Org 64, 37-44.

Cusack RR, DB Groman, SRM Jones. 2002. Rickettsial infection in farmed Atlantic salmon in eastern Canada. Can Vet J 43,435-440.

Forbes BA, DF Sahm, AS Weissfeld. 1998. Bailey \& Scott's Diagnostic Microbiology. 10 $0^{\text {th }}$ ed. Von Hoffmann Press Mosby, St. Louis, Missouri, USA, Pp 253-256.

Fryer JL, CN Lannan, LH Garcés, JJ Larenas, PA Smith. 1990. Isolation of a rickettsiales like organism from diseased coho salmon (Oncorhynchus kisutch) in Chile. Fish Pathol 25, 107-114.

Fryer JL, CN Lannan, SJ Giovannoni, ND Wood. 1992. Piscirickettsia salmonis gen. nov., sp. nov., the causative agent of an epizootic disease in salmonid fishes. Int J Syst Bacteriol 42, 120-126.

Fryer JL, RP Hedrick. 2003. Piscirickettsia salmonis: a Gram-negative intracellular bacterial pathogen of fish. J Fish Dis 26, 251-262.

Garcés LH, JJ Larenas, PA Smith, S Sandino, CN Lannan, JL Fryer. 1991. Infectivity of a rickettsia isolated from coho salmon Oncorhynchus kisutch. Dis Aquat Org 11, 93-97. 
Gómez F, V Henríquez, SH Mashall. 2009. Additional evidence of the facultative intracellular nature of the fish bacterial pathogen Piscirickettsia salmonis. Arch Med Vet 41, 261-267.

Mauel MJ, SJ Giovannoni, JL Fryer. 1996. Development of polymerase chain reaction assays for detection, identification, and differentiation of Piscirickettsia salmonis. Dis Aquat Org 26, 189-195.

Mauel MJ, DL Miller. 2002. Piscirickettsiosis and piscirickettsiosis-like infections in fish: a review. Vet Microbiol 87, 279-289.

Mauel M, C Ware, P Smith. 2008. Culture of Piscirickettsia salmonis on enriched blood agar. J Vet Diagn Invest 20, 213-214.

McCarthy U, NA Steiropoulos, KD Thompson, A Adams, AE Ellis, HW Ferguson. 2005. Confirmation of Piscirickettsia salmonis as a pathogen in European sea bass Dicentrarchus labrax and phylogenetic comparison with salmonid strains. Dis Aquat Org 64, 107-119.

McCarthy UM, JE Bron, L Brown, F Pourahmad, IR Bricknell, KD Thompson, A Adams, AE Ellis. 2008. Survival and replication of
Piscirickettsia salmonis in rainbow trout head kidney macrophages. Fish Shellfish Immunol 25, 477-484.

Mikalsen J, O Skaervik, J Wiik-Nielsen, M Wasmuth, D Colquhoun. 2008. Agar culture of Piscirickettsia salmonis, a serious pathogen of farmed salmonid and marine fish. FEMS Microbiol Lett 278, 43-47.

Olsen AB, HP Melby, L Speilberg, O Evensen, T Hastein. 1997. Piscirickettsia salmonis infection in Atlantic salmon Salmo salar in Norway-epidemiological, pathological and microbiological findings. Dis Aquat Org 31, 35-48.

Ratledge C. 2004. Iron, mycobacteria and tuberculosis. Tuberculosis 84, 110-130.

Rodger HD, EM Drinan. 1993. Observation of a rickettsia like organism in Atlantic salmon, Salmo salar L., in Ireland. J Fish Dis 16, 361-369.

Rojas V, N Galanti, N Bols, S Marshall. 2009. Productive infection of Piscirickettsia salmonis in macrophages and monocyte-like cells from rainbow trout, a possible survival strategy. J Cell Biochem 108, 631-637. 